\title{
EL DECLIVE DE LA POBOACIÓN EN GALICIA. ¿HACIA EL VACÍO DEMOGRÁFICO?
}

\author{
José Antonio Aldrey VÁZQUEZ \\ Departamento de Xeografía. Universidade de Santiago de Compostela \\ joseantonio.aldrey@usc.es \\ Xosé Constenla VEGA \\ Departamento de Didácticas Especiais. Universidade de Vigo \\ xose.constenla@uvigo.es
}

\begin{abstract}
Resumen
En este trabajo se realiza un análisis demográfico clásico de la población gallega. Además de realizar una introducción teórica sobre los estudios de población que se han realizado sobre la Comunidad Autónoma, se abordan los aspectos fundamentales que atañen a la dinámica y estructura demográfica de Galicia: su evolución y, su estructura por edad y sexo, concluyendo con la presentación de una proyección de la misma a medio plazo. Las escalas de análisis varían de la caracterización del conjunto de la comunidad a las provinciales o locales, incidiendo también en las comarcales cuando el tipo de datos existentes así lo requiere. La intención del mismo no es otra que la de constatar si realmente existe un problema demográfico, tal y como indican los medios de comunicación constantemente, y si se constata, caracterizar su gravedad y cómo evolucionará a medio plazo.
\end{abstract}

Palabras Clave: Galicia, demografía, proyecciones demográficas.

\begin{abstract}
In this paper we will make a recent demographical analysis about the development of the population in Galicia. Will also discuss fundamental aspects in relation with the dynamic and demographical structure in Galicia: the evolution and age structure; finishing off with a presentation on the medium term. The intention of this paperwork is to realise and check if there is really a demographical problem like the local media is constantly talking about. And if we find evidence, check how severe it is and how it will develop on the medium term.
\end{abstract}

Key Words: Galicia, demography, demographic projections.

\section{Los estudios demográficos sobre Galicia}

La demografía, y más concretamente la Geografía de la Población o Geodemografía, es una disciplina poco desarrollada y de reciente aparición, pues fueron muy pocos los trabajos que sobre esta temática se hicieron con anterioridad a la década de 1970 (ALDREY, 2003), y se mantuvieron en 
un número reducido hasta bien avanzada la década de 1990 o comienzos de la de 2000 (HERNÁNDEZ BORGE, 2003).

Fue a partir de esa primera fecha mencionada cuando la población gallega comenzó a contar con algunos estudios, fundamentalmente de autoría de Jean-René Bertrand, Julio Hernández Borge y Xosé Manuel Souto. El primero de ellos llegó a Galicia a mediados de los años 1970 para realizar su Tesis de Estado sobre la población gallega, publicando varios trabajos sobre esta temática previos a la finalización de esa tesis, que se tituló La population de Galice, étude géographique (1986), publicada en gallego en 1992. Por su parte, el profesor Hernández Borge es el autor que más estudios ha publicado sobre la materia, tocando en ellos diferentes temáticas dentro de las cuestiones demográficas. Por su parte, Xosé Manuel Souto se ocupó de hacer estudios globales del conjunto de Galicia, ya fuese solo (1988) o en coautoría con otros autores (CAMPILLO, MÉNDEZ Y SOUTO, 1993).

Pero no sólo la Geografía se ocupó de estudiar la población, sino que también desde otras ciencias como la historia, la economía, la sociología o la estadística aparecieron trabajos sobre demografía (FERRÁS, 2011), evidenciándose que este tipo de estudios tienen un marcado carácter pluridisciplinar (ALDREY, 2003).

A partir de 1990 destacan sobre todo trabajos que se ocupan del estado de la población de Galicia, con una preocupación creciente sobre la ya incipiente crisis demográfica que comienza a dejar patentes sus efectos sobre el territorio (HERNÁNDEZ BORGE, 1990; PRECEDO Y VILLARINO, 1994; y, RODRíGUEZ GONZÁLEZ et al., 1999).

Esta preocupación se incrementa y durante la década siguiente siguen publicándose estudios, cada vez más numerosos, en los que se siguen midiendo los impactos de una evolución demográfica cada vez más negativa (ALDREY, 2006; HERNÁNDEZ BORGE, 2005). Como la situación no mejoró, sino todo lo contrario, a partir de 2010 aparecen títulos que se preocupan ya por el negro porvenir demográfico a corto y largo plazo, y se generaliza su estudio a varias disciplinas, apareciendo expresiones como catástrofe demográfica, suicido demográfico, envejecimiento anunciado, etc. (HERNÁNDEZ BORGE, 2011; BARREIRO, PASCUAL Y MONZÓ, 2013; BLANCO DESAR, 2014; GONZÁLEZ REBOREDO, 2014; ALDREY Y DEL RÍO, 2014).

En todo caso, se puede afirmar que se observa la existencia de muchas publicaciones con contenidos teóricos, muchas veces reiterativos en la información presentada, pero muy pocos intentan ir más allá, ofreciendo soluciones para la planificación territorial o para la ayuda en el diseño de políticas públicas que puedan incidir en mitigar las consecuencias de la crisis demográfica de Galicia (ALDREY, 2003; FERRÁS, 2011).

\section{La evolución provincial y del conjunto de Galicia}

Para poder comprender bien la situación actual de la población de Galicia y apuntar cual puede ser su situación en los próximos años, se hace necesario un conocimiento de cómo ha evolucionado desde comienzos del siglo XX. 
El gran cambio demográfico en este período temporal se produce en el nivel del conjunto demográfico gallego a partir de 1960, pues hasta entonces había existido un crecimiento poblacional moderado pero continuado (CAMPILLO, MÉNDEZ Y SOUTO, 1993). A partir de esa década aparecen episodios de estancamiento, reducción y leves recuperaciones, que se han tornado ya en un descenso en todas las provincias a partir de 2011, tal y como profundizaremos algo más adelante.

La situación referida entra en contraste con el aumento continuado de la población en el conjunto de España. La repercusión inmediata de esta constatación es la pérdida de peso relativo de Galicia en lo tocante al volumen demográfico con relación al estatal. De este modo, pasó de suponer un $11 \%$ a comienzos del siglo XX a situarse en un 5,9 \% atendiendo a los datos del Padrón de Habitantes de 2018. La causa es la citada reiteradamente por los investigadores sobre demografía en Galicia (CAMPILLO, MÉNDEZ Y SOUTO, 1993; HERNÁNDEZ BORGE, 1990 у 2001; ALDREY, 2006): los continuos saldos migratorios negativos fruto de la emigración continuada (interrumpida por las prohibiciones o dificultades en las salidas en algunos períodos), que incidió en un envejecimiento progresivo de Galicia (al irse los jóvenes) y una reducción acusada de la natalidad (por la misma razón y por las dificultades económicas de los que se quedaron, además del cambio social común a toda Europa y otras regiones del globo).

Tabla 1.- Evolución de la población a nivel provincial, del conjunto de Galicia y de España, 1900-2018.

\begin{tabular}{|l|c|c|c|c|c|c|}
\hline Año & A Coruña & Lugo & Ourense & Pontevedra & Galicia & España \\
\hline $\mathbf{1 9 0 0}$ & 681.895 & 476.357 & 419.665 & 495.721 & 2.073 .638 & 18.830 .649 \\
\hline $\mathbf{1 9 1 0}$ & 726.697 & 512.467 & 446.342 & 546.247 & 2.231 .753 & 19.990 .909 \\
\hline $\mathbf{1 9 2 0}$ & 772.363 & 517.918 & 466.398 & 585.866 & 2.342 .545 & 22.012 .663 \\
\hline $\mathbf{1 9 3 0}$ & 835.906 & 523.911 & 476.336 & 630.446 & 2.466 .599 & 24.026 .571 \\
\hline $\mathbf{1 9 4 0}$ & 912.662 & 545.128 & 494.966 & 685.801 & 2.638 .557 & 26.386 .854 \\
\hline $\mathbf{1 9 5 0}$ & 971.641 & 521.213 & 494.283 & 714.666 & 2.701 .803 & 28.172 .268 \\
\hline $\mathbf{1 9 6 0}$ & 1.035 .619 & 491.955 & 488.241 & 715.181 & 2.730 .996 & 30.776 .935 \\
\hline $\mathbf{1 9 7 0}$ & 1.030 .745 & 423.064 & 441.260 & 781.334 & 2.676 .403 & 34.041 .531 \\
\hline $\mathbf{1 9 8 1}$ & 1.093 .122 & 405.377 & 430.175 & 883.268 & 2.811 .942 & 37.683 .363 \\
\hline $\mathbf{1 9 9 1}$ & 1.096 .966 & 384.365 & 353.491 & 896.847 & 2.731 .669 & 38.872 .268 \\
\hline $\mathbf{2 0 0 1}$ & 1.096 .027 & 357.648 & 338.446 & 903.759 & 2.695 .880 & 40.847 .371 \\
\hline $\mathbf{2 0 1 1}$ & 1.141 .286 & 348.067 & 328.697 & 954.877 & 2.772 .928 & 46.815 .917 \\
\hline $\mathbf{2 0 1 8}$ & 1.119 .091 & 331.232 & 309.112 & 941.535 & 2.700 .970 & 46.698 .569 \\
\hline
\end{tabular}

Fuente: Elaboración propia en base a datos de los Censos de Población, 1900-2011 (INE); y del Padrón de Habitantes (IGE), 2018. 
Esta evolución puede ser matizada si nos acercamos al comportamiento provincial y a los datos intercensales (hasta 2011) y padronales (desde 2012 hasta 2018). Un primer momento señalado por los investigadores y fácilmente comprobable en los datos de la tabla y figura 1 es el que comprende hasta 1930 (Hernández Borge, 1990; Aldrey Vázquez, 2006), cuando ya se pueden vislumbrar ritmos de crecimiento bastante más pausados en las provincias de Lugo y Ourense respecto de las atlánticas.

Si nos centramos en la evolución acontecida desde el año 2000, podemos apuntar que durante el período 2001-2011 se produce un leve repunte demográfico (se venía de una situación de declive desde 1981), protagonizado sólo por las provincias occidentales, pero con fuerza suficiente para contrarrestar la notable pérdida de efectivos que venían arrastrando desde tiempo atrás tanto Lugo como Ourense. Incidió en esta recuperación un saldo migratorio positivo, ya que se logró el asentamiento de un mayor número de foráneos que las pérdidas de gallegos hacia el exterior (la emigración de jóvenes continuó durante este período intercensal) siguiendo la dinámica de recepción de inmigrantes que vivió el conjunto del Estado, aunque en el caso que nos ocupa con una intensidad mucho menor (ALDREY Y DURÁN, 2011).

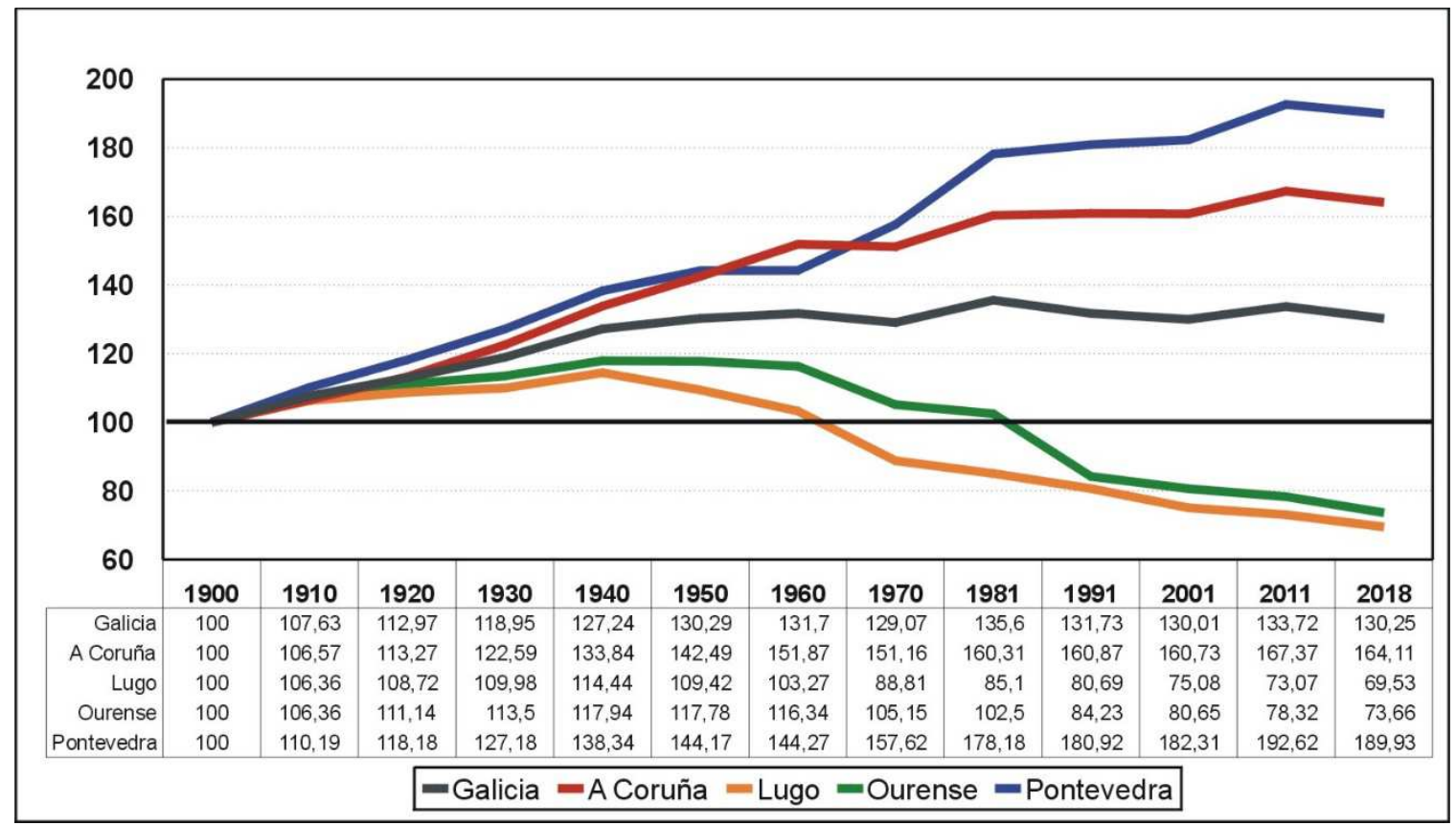

Figura 1.-Evolución demográfica de Galicia y provincias (1900-2018). 1900=100.

Fuente: Elaboración propia en base a datos de los Censos de Población, 1900-2011 (INE); y del Padrón de Habitantes (IGE), 2018.

La fuerte crisis económica iniciada en 2008 y de la que apenas estamos saliendo en el presente, es la base de la nueva caída continuada que vive Galicia. Cortados los flujos de inmigración que se habían producido en el período precedente, el saldo vegetativo negativo cada vez más intenso y la nueva emigración de jóvenes hacia el exterior ante las negativas perspectivas vitales y laborales, la población ha entrado en una senda de caída libre que por primera vez incluye también a la provincia de Pontevedra, que durante todo el período considerado había conseguido incrementar su 
población. La situación de pérdida de efectivos parece ahora una situación francamente difícil de revertir, porque como veremos a continuación, está llevando a muchas áreas de Galicia hacia una progresiva desertización.

\section{La evolución demográfica a escala municipal}

Los escasos matices que ofrece la visualización de los datos a una escala provincial como la que acabamos de hacer, se pueden enriquecer con un análisis en el que introduzcamos una cartografía a escala municipal.

El intervalo temporal que utilizaremos en esta aproximación será para los veinte años transcurridos entre 1998 y 2017. La figura 2 muestra la evolución demográfica municipal ocurrida entre esas dos fechas. En ella apreciamos unas pérdidas demográficas sumamente notables en una gran parte del territorio gallego. Más de la mitad de sus municipios (164 de 315, el $52 \%$ ) perdieron más del $20 \%$ de su población, y aún podemos matizar más indicando que 54 ayuntamientos tuvieron pérdidas superiores a un tercio de su población (17\%), llegando a casos extremos como los de Dozón, San Xoán de Río, Chandrexa de Queixa, A Teixeira, Agolada, Muras, Navia de Suarna, Montederramo, Rodeiro, Beariz, Ribeira de Piquín o Covelo, en los que en estos veinte años han perdido por encima del $45 \%$ de su población.

En el mapa apreciamos como una grandísima parte del territorio oriental de la Comunidad Autónoma, en especial las zonas de topografía más accidentada, como son los municipios de montaña de Lugo y Ourense, aquéllos limítrofes entre estas provincias y las atlánticas, espacios con comunicaciones deficientes (como en el área centro occidental coruñesa, Ortegal, Costa da Morte), ha tenido una muy fuerte caída demográfica, que se viene a sumar a la que ya habían vivido a lo largo de buena parte del siglo XX (CAMPILLO, MÉNDEZ Y SOUTO, 1993; ALDREY, 2006). Fuera de estos espacios con pérdidas tan notables, hemos de indicar que la caída demográfica se ha producido en 243 municipios (un $77 \%$ del total), escapando a ella únicamente pequeñas porciones del territorio.

Los espacios que presentan un crecimiento del número de habitantes desde 1998 ocupan un territorio exiguo, con una clara concentración en las áreas periurbanas de las principales ciudades de Galicia, donde se dieron los incrementos más notables, llegando a superar el $20 \%$ en municipios periféricos a las siete ciudades, en los que se han vivido intensos procesos de desconcentración demográfica y económica desde la urbe inmediata (son tan solo 25 municipios, el $8 \%$ ) y el único que está fuera de la órbita de las ciudades es Burela, en la Mariña lucense. Algunos de ellos han tenido crecimientos ciertamente espectaculares, destacando por encima de todos Ames y Barbadás (crecimientos del $117 \%$ y $93 \%$, respectivamente), aunque varios de ellos tienen por encima del $50 \%$ más de población que en 1998 o rozan ese incremento (Culleredo, Oroso, San Cibrao das Viñas, Salceda de Caselas, Cambre, Arteixo, Sada u Oleiros). Fuera de esas áreas urbanas sólo aparecen con valores positivos algunas pequeñas ciudades ("vilas"), buena parte de la costa de las Rías Baixas y algunos municipios de la Mariña lucense. Dos ciudades, Ferrol y Ourense no han conseguido crecer 
en estos veinte años, la primera por la crisis económica profunda en la que está sumida desde hace ya tiempo y, en el caso de la segunda, por el declive demográfico acusado de la provincia que centraliza y los fuertes procesos de desconcentración residencial que ha vivido hacia los municipios situados hacia el sur y este de la urbe, que han favorecido esta situación. El resto de ciudades, excepto Pontevedra y Lugo con incrementos relativamente importantes, han conocido un leve progreso demográfico, influidas también por los notables crecimientos de sus periferias.

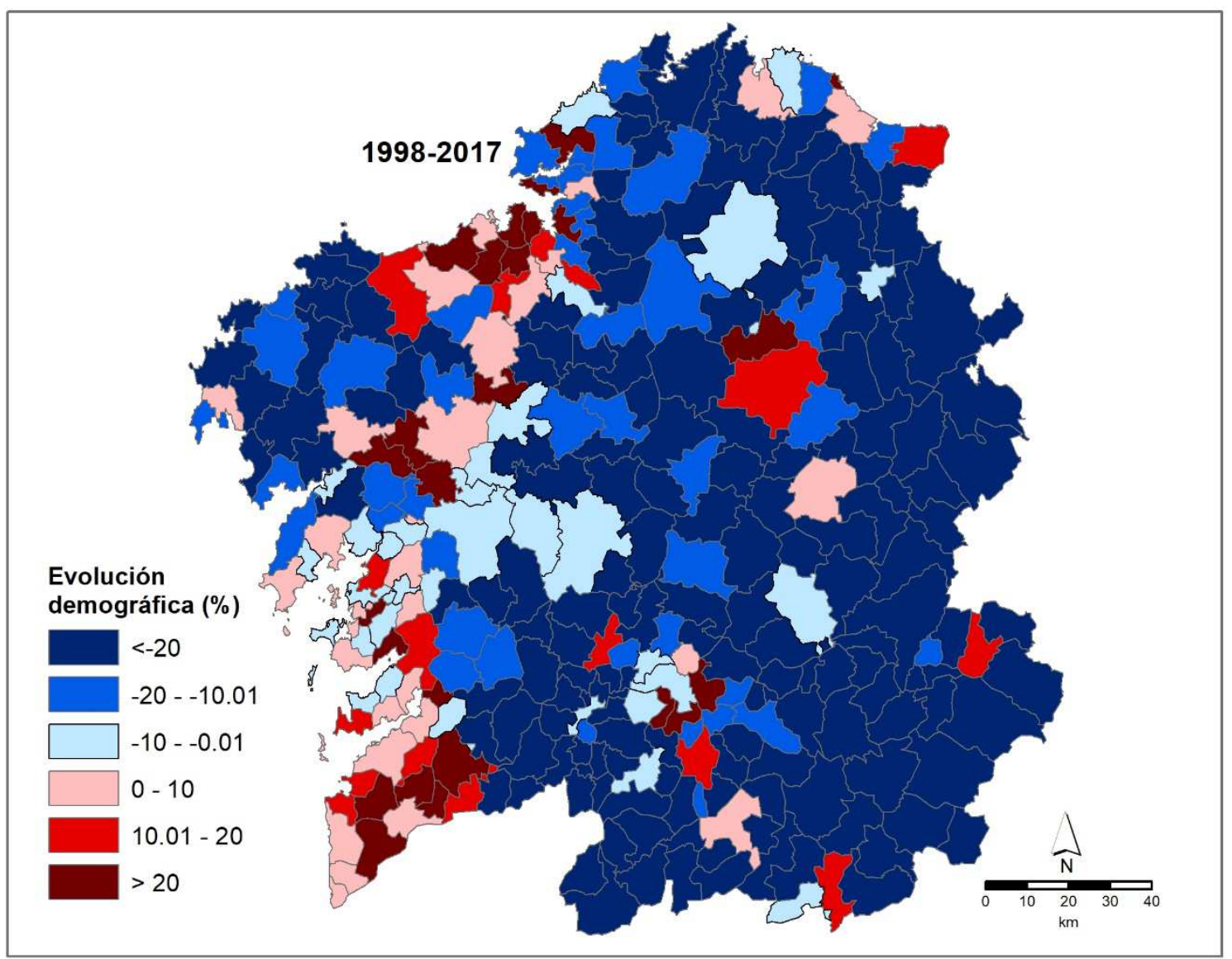

Figura 2.- Evolución demográfica municipal. 1998-2017.

Fuente: Elaboración propia en base a datos de la estadística Padrón Municipal de Habitantes. IGE.

Otros datos reveladores de como el aumento demográfico se concentra en determinadas áreas de las provincias de A Coruña y Pontevedra son que el $28,7 \%$ y el $46,8 \%$ de sus municipios, respectivamente, crecieron durante el período (27 y 29 ayuntamientos). Frente a un 10,4\% de los municipios lucenses ( 7 en números absolutos) y un $9,8 \%$ de los ourensanos ( 9 en total).

Podemos concluir indicando que el crecimiento se produce principalmente en el eje atlántico y en cualquier caso es urbano (en un sentido amplio, de áreas urbanas) y/o litoral. Por otra parte, todos los datos disponibles parecen indicar que el proceso de despoblación es acumulativo: cada vez existe menos población y progresivamente se pierden más efectivos demográficos. Cuanta menor población queda en un municipio este tiende a despoblarse más rápidamente. $Y$ ello incide también en un menor crecimiento de las áreas más dinámicas que se restringen progresivamente. 


\section{La estructura por edad}

Vista la evolución de la población en Galicia la estructura por edad es otro de los vectores fundamentales para poder hacer una caracterización de la demografía de Galicia.

Tabla 2.- Estructura por grandes grupos de edad (\%). 2002 y 2017

\begin{tabular}{|l|r|r|r|r|r|r|}
\hline \multicolumn{1}{|c|}{ Edad } & \multicolumn{2}{|c|}{$<16$} & \multicolumn{2}{c|}{$16-64$} & \multicolumn{2}{c|}{$65 \mathrm{y}+$} \\
\hline \multicolumn{1}{|c|}{ año } & $\mathbf{2 0 0 2}$ & $\mathbf{2 0 1 7}$ & $\mathbf{2 0 0 2}$ & $\mathbf{2 0 1 7}$ & $\mathbf{2 0 0 2}$ & $\mathbf{2 0 1 7}$ \\
\hline Galicia & 12,83 & 12,76 & 66,09 & 62,67 & 21,08 & 24,57 \\
\hline A Coruña & 12,64 & 13,00 & 67,50 & 63,05 & 19,86 & 23,95 \\
\hline Lugo & 11,08 & 10,61 & 61,47 & 60,66 & 27,45 & 28,73 \\
\hline Ourense & 10,98 & 10,49 & 61,11 & 58,58 & 27,91 & 30,93 \\
\hline Pontevedra & 14,45 & 13,98 & 68,07 & 64,30 & 17,49 & 21,73 \\
\hline España & 15,60 & 16,03 & 67,39 & 65,01 & 17,01 & 18,96 \\
\hline
\end{tabular}

Fuente: Elaboración propia en base a datos de la estadística Cifras poboacionais de referencia. IGE.

Si nos fijamos en los datos (tabla 2), vemos como desde 2002 hasta la actualidad la situación de los diferentes grupos de edad ha variado de manera notable. Así, Galicia ha visto acrecentar de significativamente su índice de envejecimiento, pasando de poco más de una quinta parte de su colectivo demográfico a casi uno de cada cuatro efectivos. Sin embargo, el grupo de jóvenes ha mantenido cierta estabilidad, marcado por una pequeña recuperación del número de nacimientos en este período, tal y como ocurrió en el resto de España, aunque la tendencia de los últimos datos existentes marca una nueva bajada de la natalidad. De esta manera, el grupo de viejos se ha estado nutriendo básicamente del de adultos, que ha visto bajar su proporción en la misma medida que se ha incrementado el de la población anciana. Esto supone una cierta novedad, porque como han apuntado otros estudios (Aldrey, 2006), el grupo de adultos fue el que a lo largo de todo el siglo XX se mantuvo más estable, porque se nutría el de viejos al mismo tiempo que se reducía la proporción de jóvenes, aunque con más efectivos cada vez entre los 40 años. El fuerte envejecimiento actual y la marcha de parte de los efectivos jóvenes dentro de este grupo hacia la emigración (como vimos) hacen que se esté reduciendo su proporción de manera acusada y que esto repercuta fundamentalmente en el de personas más mayores. Por provincias, como cabría esperar por los aspectos ya vistos hasta el momento, son Pontevedra y A Coruña (por este orden) las que cuentan con una mayor proporción de personas jóvenes (Pontevedra destaca, con un grupo de jóvenes del 14 $\%$, frente a A Coruña con un punto menos), aunque se sitúan claramente por debajo de la media estatal (tabla 2). Son también las provincias menos envejecidas, aunque lejos de la media estatal. En este aspecto, el del envejecimiento, el progreso del índice en los 15 años que separan las dos fechas 
contempladas ha sido muy acusado en todo el territorio gallego, aunque el caso de Ourense es especialmente significativo con casi un tercio de su población por encima de los 65 años.

Estos datos a nivel provincial, sin duda significativos, no permiten matizar la magnitud del envejecimiento que estamos viendo y no indican donde aparecen las mayores proporciones o donde resulta menos grave. Para poder comprenderlo mejor se hace necesario descender a una escala de análisis municipal (figura 3).

En lo que respecta a la distribución de los menores de 16 años (figura 3, superior), 244 municipios (el $77 \%$ ) están por debajo de la media gallega del $12,76 \%$ de personas de esa edad. Por debajo del $10 \%$ se sitúan 188 municipios (el $60 \%$ ), abarcando la práctica totalidad de Lugo y Ourense (sólo escapan a esa cifra algunas "vilas", las capitales provinciales y algunos de sus municipios periurbanos) y la gran mayoría del territorio de las provincias atlánticas, huyendo de esa dinámica únicamente el denominado Eje Urbano Atlántico (Pazos, 2005), ampliado a buena parte de la costa al sur de Ribeira y algunas pequeñas ciudades como Lalín o Melide. Este espacio, como vimos en su momento, es el que ha concentrado también los crecimientos demográficos y el asentamiento de los inmigrantes, de manera que se convierte cada vez más en el baluarte demográfico de Galicia, sin indicadores de gran vitalidad, pero mucho mejores que los del resto del territorio. Un ejemplo de ello es que sólo 18 municipios gallegos (6\% del total), todos de las orlas periurbanas de las ciudades, presentan un grupo de menores de 16 años que supere el $15 \%$ de su colectivo demográfico. Por tanto, podemos concluir que los jóvenes escasean en todo el territorio, y los pocos que hay son mayoritariamente urbanos y residen en las periferias de las principales ciudades.

Si cartografiamos la proporción de mayores de 65 años (figura 3, inferior), apreciamos casi un negativo fotográfico de la situación que acabamos de describir.

Una primera visual de los colores del mapa ya nos muestra como la provincia de Ourense es la que tiene los valores más altos de municipios con envejecimientos muy elevados, al igual que Lugo, aunque en este caso cuenta con menos entidades municipales en los valores más extremos. En todo caso, en el mapa se puede señalar que cuentan con un envejecimiento superior al $30 \%$ un total de 192 municipios (61\%), cifra que ha crecido significativamente desde 2004, cuando eran 154 (el $49 \%$ ) los territorios municipales que superaban esa cifra (Aldrey, 2006). Entre ellos están buena parte de los de las provincias orientales (sólo escapan a esa cifra 14 municipios de Lugo y 12 de Ourense).

Este extremado grado de envejecimiento afecta también en buena medida a las provincias occidentales, sobre todo en las estribaciones de la Dorsal Meridiana, especialmente en la comarca de Ortegal y la parte oriental de la ferrolana, al este de la de Betanzos, a la Terra de Melide, la Terra de Soneira, Xallas, Barcala, Tabeirós-Terra de Montes, oriente de Pontevedra y la Paradanta.

Dentro de este grupo, el envejecimiento aún es mucho más grave, superando el $40 \%$ del total demográfico municipal (78 municipios, el $25 \%$ del total de Galicia), en áreas del Norte de Lugo (Ourol, Muras o Abadín) y del extremo montañoso oriental, uno coruñés (Aranga) y otro pontevedrés (Forcarei), así como en la Ulloa, el valle del Sil y buena parte de las comarcas ourensanas de $\mathrm{O}$ Carballiño, Terra de Caldelas, Allariz-Maceda, Terra de Trives, Viana, Verín, A Limia, Baixa Limia, Terra de Celanova y Valdeorras, llegando en esta provincia a haber ocho municipios que superan el 
50 \% de su población por encima de la edad de jubilación: O Bolo, Calvos de Randín, Gomesende, O Irixo, Lobeira, San Xoán de Río, A Teixeira y Verea

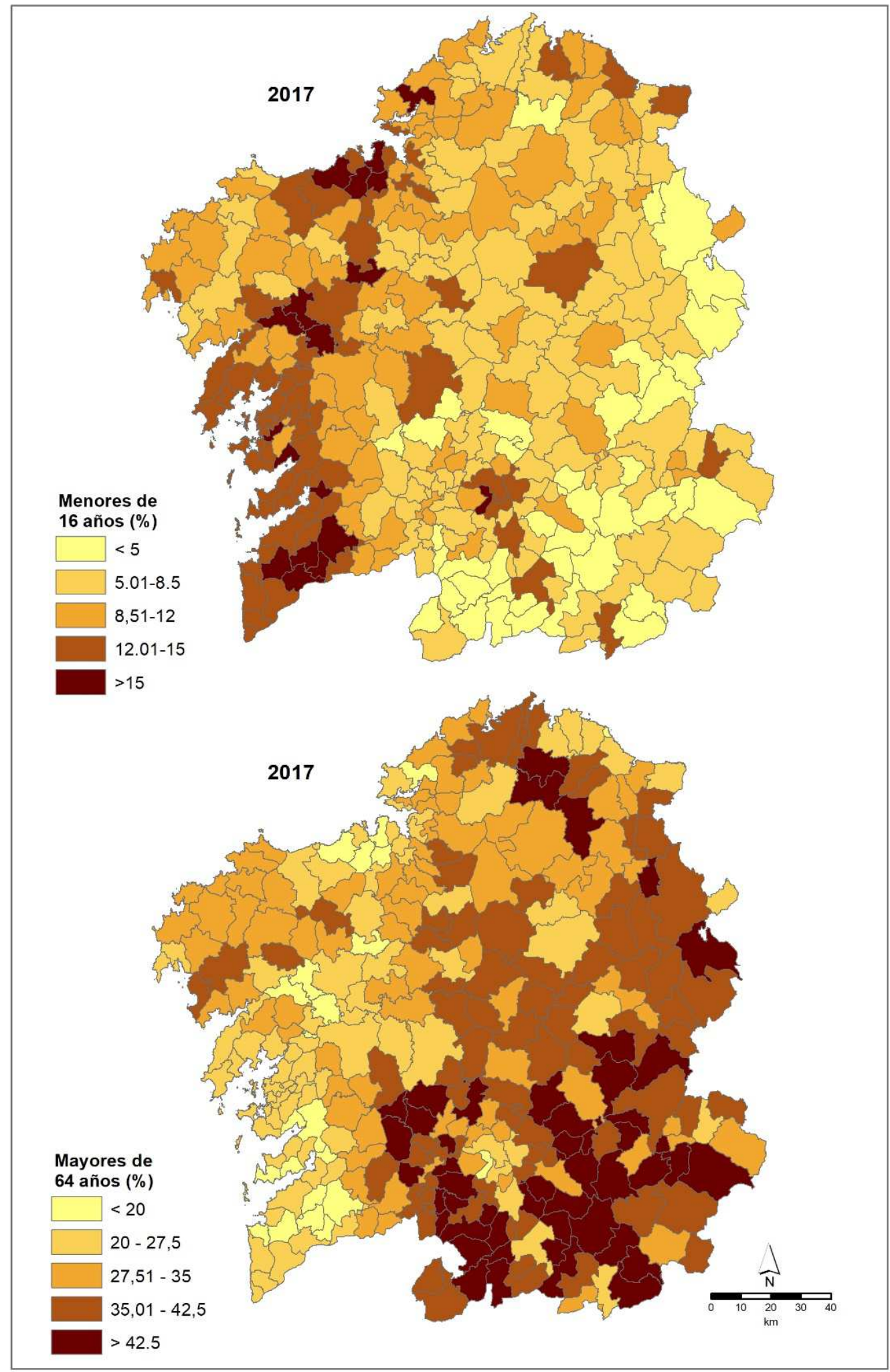

Figura 3.- Menores de 16 años y mayores de 65 años a escala municipal, 2017.Fuente: Elaboración propia en base a datos del Padrón de Habitantes. IGE. 
Por su parte, los municipios con menores contingentes de personas mayores son los mismos que contaban con la mayor proporción de jóvenes, es decir, los de las periferias de las principales ciudades del Eje Atlántico y en general en los municipios urbanos y costeros de las Rías Baixas, así como en las principales "vilas". Son estos espacios, muy limitados en extensión en el global de Galicia, los que cuentan como venimos reiterando con las mejores perspectivas demográficas.

\section{Las proyecciones demográficas a medio plazo}

Para tener una idea de cuál puede ser la evolución a medio plazo utilizaremos las proyecciones publicadas recientemente por el IGE (con fecha de referencia de los datos de 1 de enero de 2015), con un horizonte que abarca hasta el 2031 (15 años desde que se realizaron, trece ahora en 2018). Buscando una fiabilidad aceptable el organismo estadístico de Galicia (IGE) ha realizado estas proyecciones a una escala comarcal, debido a los escasos volúmenes demográficos con los que cuentan muchos de los municipios de Galicia.

De este modo, las proyecciones consultadas publicadas por el IGE nos muestran como las tendencias apuntadas de descenso demográfico se confirman, con una caída que, para el conjunto de Galicia y hasta el 2031, se acercaría a un $8 \%$, con unas pérdidas de más de 210.000 habitantes, para contar con una población inferior a los 2,5 millones de efectivos en esa fecha (tabla 3).

Tabla 3.- Proyección demográfica Galicia y provincias, 2031

\begin{tabular}{|l|r|r|r|r|}
\hline Año & \multicolumn{1}{|c|}{$\mathbf{2 0 1 8}$} & \multicolumn{1}{c|}{$\mathbf{2 0 3 1}$} & Evolución & \% \\
\hline Galicia & 2.700 .970 & 2.489 .946 & -211.024 & $-7,81$ \\
\hline A Coruña & 1.119 .091 & 1.038 .034 & -81.057 & $-7,24$ \\
\hline Lugo & 331.232 & 298.151 & -33.081 & $-9,99$ \\
\hline Ourense & 309.112 & 275.094 & -34.018 & $-11,01$ \\
\hline Pontevedra & 941.535 & 878.668 & -62.867 & $-6,68$ \\
\hline
\end{tabular}

Fuente: Elaboración propia en base Proxeccións de Poboación. IGE. Dato de 2018 tomado del Padrón de Habitantes, IGE.

Las pérdidas serían notables en las cuatro provincias, si bien estas serían menores en Pontevedra y A Coruña (circunstancia lógica dada su menor senectud y el comportamiento no tan negativo de su saldo natural) y francamente acusadas en Lugo y especialmente en Ourense, con un $10 \%$ de descenso en el primer caso y por encima del $11 \%$ en el segundo, de manera que ambas provincias bajarían decididamente de los 300.000 habitantes (tabla 3).

Si cartografiamos a nivel comarcal la evolución demográfica hasta 2031 (figura 4), podemos comprobar como con la única excepción de la comarca de Santiago, todas ellas verán descender su población, algunas de forma muy acusada, lo cual vendría a agravar la situación de espacios ya 
francamente en declive como vimos en los capítulos previos de este trabajo. Así, comarcas de las sierras orientales, del sur y centro ourensano, y las coruñesas de Ortegal, Muros y Terra de Soneira, conocerán descensos superiores al $20 \%$. En el extremo opuesto, además de la ya citada de Santiago con valores positivos, sólo aparecen comarcas urbanas (las centralizadas por las dos capitales del interior, A Coruña, Pontevedra) y A Paradanta, con caídas demográficas poco acusadas. En todo caso el panorama es francamente negativo e incidirá en un claro agravamiento de los problemas de despoblación y envejecimiento de amplias áreas de Galicia.

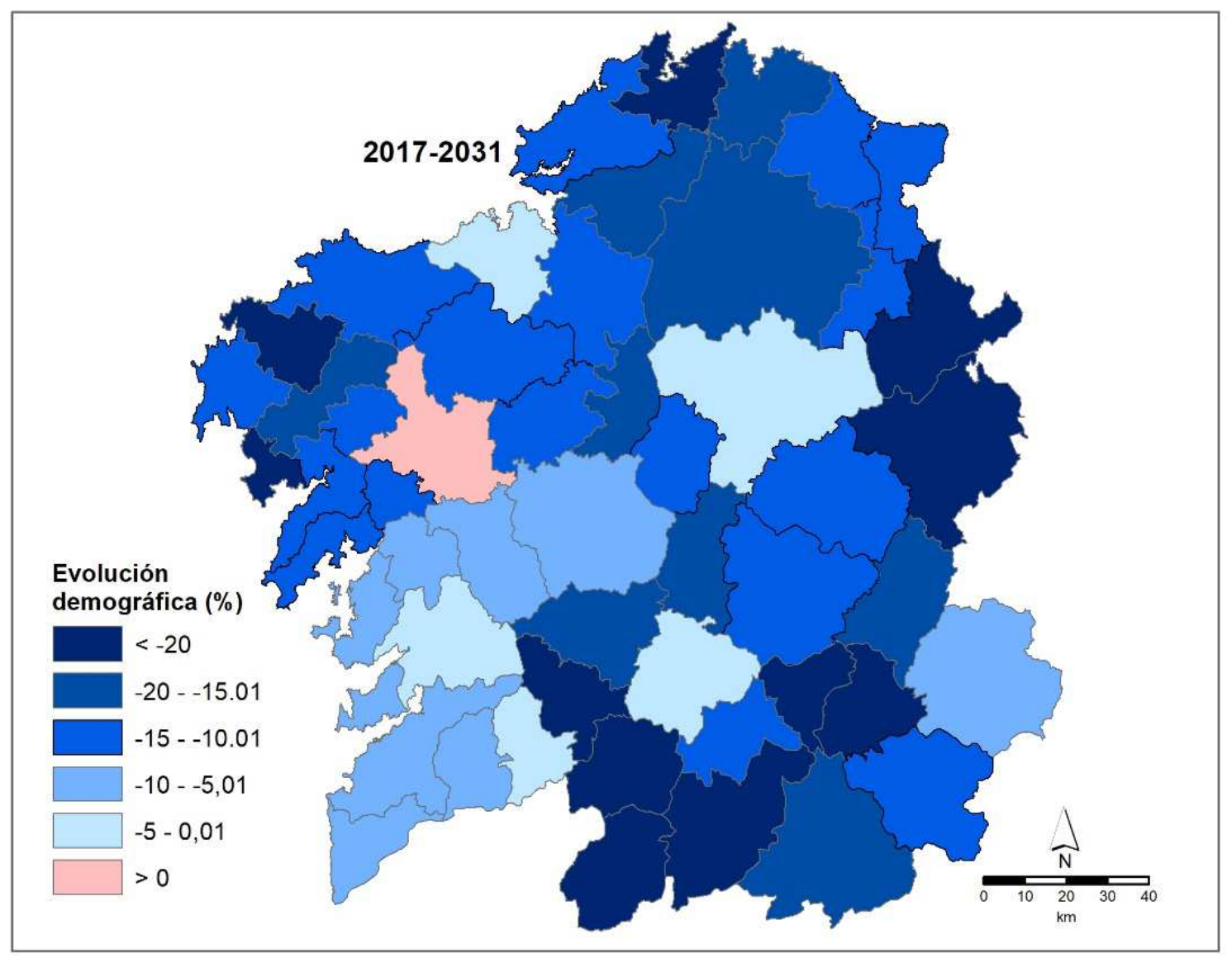

Figura 4.-Evolución demográfica comarcal, 2017-2031.

Fuente: Elaboración propia en base Proxeccións de Poboación. IGE. Datos de 2017 tomados del Padrón de Habitantes, IGE.

Por tanto, lo que marcará la evolución demográfica y de las densidades es un mantenimiento de las disparidades actuales, acentuándose aún más, con una clara dualidad entre los espacios litorales (con la excepción del Ortegal y la Costa da Morte al norte de Fisterra), urbanos (incluyendo las principales "vilas") y periurbanos, con respecto al resto del territorio en franco declive demográfico y con una tendencia clara hacia la despoblación que se ve muy claramente en la disminución de las densidades demográficas (Aldrey et al., 2013), con áreas concretas que prácticamente serán a la altura de 2030 espacios semidespoblados. 


\section{Conclusión}

Tras el análisis realizado podemos concluir que sí que parece que Galicia camine hacia una desertización demográfica, pues cualquier política demográfica que se quiera poner en práctica chocará de frente con el extremado envejecimiento, difícilmente reversible, aunque pudiesen "inyectarse" efectivos jóvenes en la estructura por edades. Algo que no parece factible, pues aunque el saldo migratorio se mantenga en valores próximos a cero o positivos, la salida de personas jóvenes está siendo muy importante y dificulta cualquier atisbo de recuperación. Desde luego, la única salida parece la llegada de inmigrantes jóvenes, dispuestos a tener descendencia en Galicia, pero esta posibilidad es desde luego lejana pues la manera de atraerlos sería con una oferta laboral atractiva y estable, aspectos ambos que no se dan ni parece que se vayan a dar en un futuro próximo.

En todo caso podemos constatar, una vez realizado el estudio, que en un futuro a medio plazo se va a mantener la dualidad entre una Galicia urbana, "vilega" y costera (con la excepción de la comarca de Ortegal y del tramo coruñés entre Cee y Carballo), que se concentra en menos de un tercio del territorio, y una Galicia oriental y del interior. Diferencia que será cada vez más acusada, de manera que la Galicia algo más joven y dinámica continuará densificándose en detrimento del resto del territorio que tendrá cada vez una población más envejecida y en franco retroceso.

En consecuencia, si tenemos una Galicia con dos realidades contrapuestas, habrá que aplicar medidas diferentes en cada uno de esos ámbitos. En el caso de la Galicia más dinámica serían necesarias políticas integradas en las áreas urbanas, superando las lógicas municipales, con dotaciones de servicios y equipamientos de carácter supramunicipal buscando lógicas sostenibles de organización territorial, económica, de movilidad, entre otras.

Por su parte, en la Galicia más regresiva, con una despoblación que crece de manera rápida y acusada, serán necesarias fórmulas de cooperación intermunicipal para hacer viables los municipios, cada vez con menor dinamismo y recursos decrecientes para hacer frente a necesidades mayores en el ámbito asistencial.

En cualquier caso, ambas lógicas no deben perder de vista la necesidad de establecer algunas líneas de actuación que entiendan el territorio en su conjunto, evitando así fenómenos de competencia interterritorial. La existencia de una Galicia dual debe hacer reflexionar sobre su conveniencia y, en su caso, sobre su superación hacia modelos de planificación y gestión que impliquen cierta corresponsabilidad espacial.

El extremado envejecimiento hará crecer las demandas de dependientes, sobre todo en áreas de accesibilidad más deficiente y tendentes a la despoblación. Esta situación no es exclusiva de Galicia sino común a la mayoría de los ámbitos rurales de Europa occidental. El éxodo rural conlleva, más tarde o más temprano, al desplome demográfico, y con él se empuja a la práctica desaparición a muchas aldeas y parroquias, así como a una pérdida de peso económico de las cabeceras comarcales que les dan cobertura terciaria, y por supuesto al paulatino desfiguramiento y posterior desaparición de hábitats y paisajes muchas veces centenarios que se explicaban y mantenían con las 
lógicas pasadas de poblamiento y aprovechamiento económico del rural, hoy desaparecidas. Esto es una consecuencia de la evolución social del proceso de urbanización, de los cambios en las pautas de vida y de los intereses de la población occidental en general, y de la gallega en particular (LOIS GONZÁLEZ, 1993 y 1996).

En este sentido, esta pérdida brusca e irreversible de contingentes demográficos provoca un deterioro importante de las relaciones sociales que construyen y dan sentido al territorio $\mathrm{y}$, en consecuencia, una pérdida de su complejidad espacial, llegando a un estado sistémico de colapso (CONSTENLA, 2018). De algún modo, el declive demográfico puede ser entendido así, como un síntoma de una patología espacial de mayor envergadura y profundidad que interpela al conjunto del territorio como un organismo con dinámicas propias.

Esta situación hace que debamos acostumbrarnos a un territorio que tendrá extensiones más o menos amplias con bajas densidades y con población muy envejecida. Con esta realidad cabe demandar a los poderes públicos, la aplicación de políticas públicas orientadas hacia las personas mayores, con provisión de cuidados en el ámbito familiar (teleasistencia, servicios de ayuda a domicilio, etc.), fundamentales para fijar a la población anciana, para que si no lo desea no tenga que abandonar su hogar para trasladarse a residir con sus familias o a residencias geriátricas, muchas veces situadas en ámbitos urbanos (ALDREY, 2009). Esto posibilitaría, además, fijar alguna población gracias a la creación de empleo relacionado con los servicios sociales y asistenciales.

Por otro lado, sería fundamental mejorar la accesibilidad hospitalaria y a los centros de salud (reducción de los tiempos de desplazamiento y mayores facilidades para acudir a las consultas), cuestiones clave para una población anciana que demanda este tipo de servicios con frecuencia.

Se trataría, en definitiva, de dotar de unos servicios sanitarios y de asistencia lo menos diferentes posible a los que las personas mayores pueden acceder en ámbitos urbanos o desde sus proximidades.

\section{Bibliografía}

ALDREY VÁZQUEZ, J. A. (2003). Xeografía Social e da Poboación na metade sur da provincia de A Coruña: unha análise a diferentes escalas. Santiago de Compostela: Universidade de Santiago de Compostela.

ALDREY VÁZQUEZ, J. A. (2006). A poboación galega, 1900-2005. Cambio demográfico e implicacións territoriais. Vigo: Ir Indo.

ALDREY VÁZQUEZ, J. A. (2009). Implicaciones socioterritoriales de la despoblación en la montaña astur-galaico-leonesa. In, L. López Trigal et al (Coords.), Envejecimiento, despoblación y territorio (pp. 547-566). León: Universidad de León.

ALDREY VÁZQUEZ, J. A. y DURÁN VILLA, F. R. (2011). As novas tendencias na poboación galega: da emigración á inmigración. In, M. J. Piñeira y X. M. Santos (Coords.), Xeografía de Galicia (pp. 229-277). Edicións Xerais de Galicia, Vigo.

ALDREY VÁZQUEZ, J. A.; FERNÁNDEZ CASAL, R.; y, LÓPEZ GONZÁLEZ, A. (2013). Proxeccións demográficas de Galicia 2011-20130. Análise dos resultados. Documento de Traballo do IDEGA, Análise Económica 55 (monográfico). 
ALDREY VÁZQUEZ, J. A. y DEL RÍO FRANQUEIRA, D. (2014). Crónica de un envejecimiento anunciado: Galicia 2015-2030. In VV.AA., Cambio demográfico y socio-territorial en un contexto de crisis. XIV Congreso Nacional de la Población Española (pp. 69-82). Sevilla: Universidad Pablo de Olavide.

BARREIRO RIVAS, X. L.; PASCUAL MONZÓ, R. M.; Y, ARAUJO CARDALDA, L. X. (Eds.). (2013). O reto demográfico de Galicia. Santiago de Compostela: Escola Galega de Administración Pública.

BERTRAND, J.-R. (1992). A Poboación de Galicia. Estudios Xeográficos. Santiago de Compostela: Universidad de Santiago de Compostela.

BLANCO DESAR, M. (Ed.). (2014). Galicia: un pobo con futuro? O noso devalo demográfico. Vigo: Edicións Xerais de Galicia.

CAMPILLO RUIZ, A.; MÉNDEZ MARTíNEZ, G.; y, SOUTO GONZÁLEZ, X. M. (1993). A Poboación e a Acción Xeodemográfica. In A. Pérez Alberti (Dir.), Xeografía de Galicia, tomo 4. Santiago de Compostela: Gran Enciclopedia Gallega.

CONSTENLA VEGA, X. (2018). O colapso territorial em Galiza. Unha ollada espacial da construción social do país. Vigo: Editorial Galaxia.

FERRÁS SEXTO, C. (2011). De la aldea al suburbio. Cambios demográficos y territoriales en Galicia, 1990-... Revista Galega de Economía, 20, 1-19.

GONZÁLEZ REBOREDO, X. (2014). Contra o suicidio demográfico polas súas consecuencias socioculturais. In, M. Blanco Desar (Ed.): Galicia un pobo con futuro? O noso devalo demográfico (pp. 13-18). Vigo: Edicións Xerais de Galicia.

HERNÁNDEZ BORGE, J. (1990). Tres millóns de galegos. Santiago de Compostela: Universidade de Santiago de Compostela.

HERNÁNDEZ BORGE, J. (2001). Los recursos demográficos. In, Ma. P. de Torres Luna (Dir.), Xeografía de Galicia. Vigo: Faro de Vigo.

HERNÁNDEZ BORGE, J. (2003). Bibliografía geodemográfica de Galicia. In, VV.AA., La dinámica geodemográfica protagonista del territorio. Actas del VIII Congreso de la Población Española (pp. 103-119). Santiago de Compostela: Universidade de Santiago de Compostela.

HERNÁNDEZ BORGE, J. (2011). Despoblación y envejecimiento: Galicia hacia el año 2020. Revista Galega de Economía, 20, Pp. 121-140.

LOIS GONZÁLEZ, R. C. (1993). Problemas para a delimitación dos espacios urbanos e rurais. In, VV.AA., Concepcións espaciais e estratexias territoriais na Historia de Galicia (pp. 201-221). Santiago de Compostela: ASGH.

LOIS GONZÁLEZ, R. C. (1996). As novas formas de urbanización. In, VV.AA., A guerra en Galicia. O rural e o urbano na Historia de Galicia (pp. 451-489). Santiago de Compostela: ASGH.

PRECEDO LEDO, A. y VILLARINO PÉREZ, M. (1994). A poboación de Galicia. A crise demográfica e o seu impacto territorial. Santiago de Compostela: Gabinete de Planificación e Desenvolvemento Territorial, Xunta de Galicia.

RODRíGUEZ GONZÁLEZ, R. et Al. (1999). A despoboación en Galicia: preocupación ou lóxica territorial. Santiago de Compostela: Tórculo. 